\title{
$J_{A W, W A}$ functions in Passarino-Veltman reduction
}

\author{
D. Bardin, L. Kalinovskaya, V. Kolesnikov and W. von Schlippe
}

November 6, 2018

\begin{abstract}
In this paper we continue to study a special class of Passarino-Veltman functions $J$ arising at the reduction of infrared divergent box diagrams. We describe a procedure of separation of two types of singularities, infrared and mass singularities, which are absorbed in simple $C_{0}$ functions. The infrared divergences of $C_{0}$ 's can be regularized then by any method: photon mass, dimensionally or by the width of an unstable particle. Functions $J$, in turn, are represented as certain linear combinations of the standard $D_{0}$ and $C_{0}$ Passarino-Veltman functions. The former are free of both types of singularities and are expressed as explicit and compact linear combinations of logarithms and dilogarithm functions. We present extensive comparisons of numerical results with those obtained with the aid of the LoopTools package.
\end{abstract}




\section{Introduction}

In the standard Passarino-Veltman reduction [1] of 4-point box functions with an internal photon line connecting two external lines on the mass shell there appears an infrared and mass singular $D_{0}$ function (see, for example, [2]). A typical example of these diagrams arising in the calculation of one-loop EW corrections to $f \bar{f} \rightarrow Z Z(Z A)$ processes was considered in [3, where a universal approach to the calculation of such diagrams was proposed.

In this paper we describe how this approach works for $t \rightarrow b f_{1} \bar{f}_{1}^{\prime}$ and $f_{1} \bar{f}_{1}^{\prime} \rightarrow t \bar{b}\left(f_{1}\right.$ is a massless fermion) Charged Current (CC) processes. For these processes one meets eight such box functions, four direct and four cross ones. Cross boxes are trivially derived from direct ones by a permutation of arguments. Boxes for $\bar{t}$ decays are related to those of $t$ decays, see Ref. [4. So, it is sufficient to consider only one pair of boxes shown in Fig. 1.
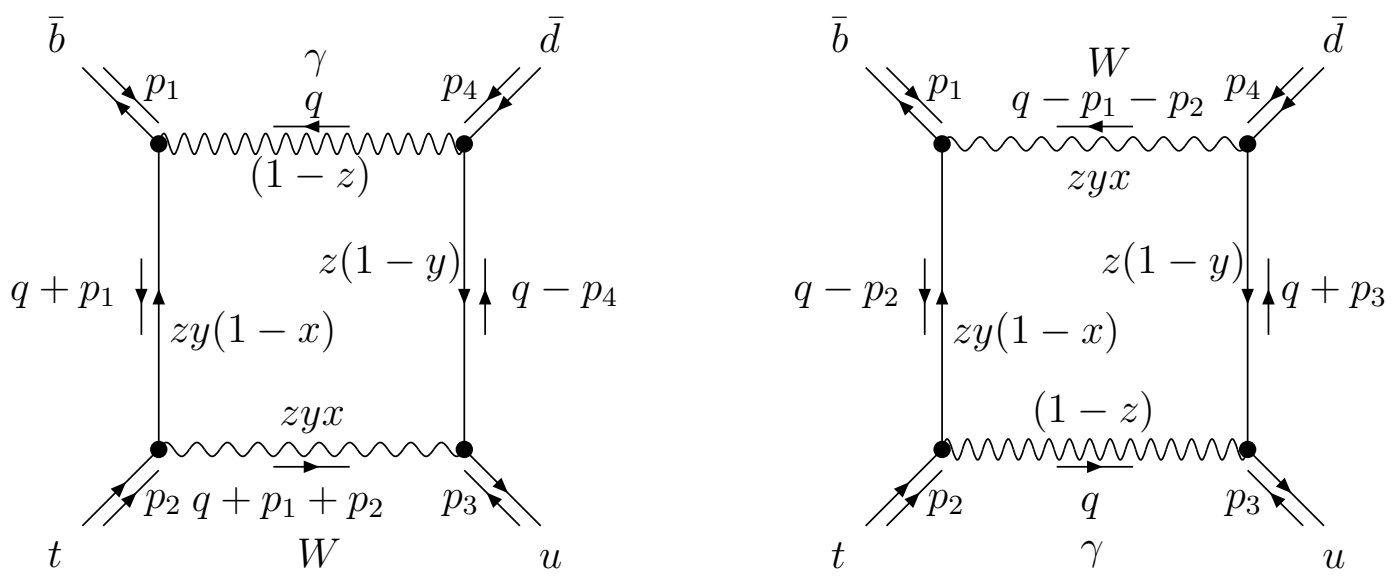

Figure 1: Remaining $J_{A W}^{d}$ (left) and $J_{W A}^{d}$ (right) functions.

Using the standard Passarino-Veltman reduction it is possible to derive relations between infrared and mass singular functions $D_{0}\left(-m_{b}^{2},-m_{t}^{2},-m_{u}^{2},-m_{d}^{2}, Q^{2}, T^{2} ; 0, m_{b}, M_{W}, m_{d}\right)$ and $C_{0}\left(-m_{d}^{2},-m_{b}^{2}, T^{2} ; m_{d}, 0, m_{b}\right)$ and an infrared finite but mass-singular auxiliary function $J_{A W}^{d}\left(Q^{2}, T^{2} ; m_{b}, m_{t}, m_{d}, m_{u}, M_{W}\right)$ and another $C_{0}\left(-m_{u}^{2},-m_{d}^{2}, Q^{2} ; M_{W}, m_{d}, 0\right)$ with mass singularity. These basic relations, exact in masses, are:

$$
\begin{aligned}
& J_{A W}^{d}\left(Q^{2}, T^{2} ; m_{b}, m_{t}, m_{d}, m_{u}, M_{W}\right)= \\
& \quad\left(M_{W}^{2}+Q^{2}\right) D_{0}\left(-m_{b}^{2},-m_{t}^{2},-m_{u}^{2},-m_{d}^{2}, Q^{2}, T^{2} ; 0, m_{b}, M_{W}, m_{d}\right) \\
& +C_{0}\left(-m_{u}^{2},-m_{d}^{2}, Q^{2} ; M_{W}, m_{d}, 0\right)-C_{0}\left(-m_{d}^{2},-m_{b}^{2}, T^{2} ; m_{d}, 0, m_{b}\right), \\
& J_{W A}^{d}\left(Q^{2}, T^{2} ; m_{t}, m_{b}, m_{u}, m_{d}, M_{W}\right)= \\
& \left(M_{W}^{2}+Q^{2}\right) D_{0}\left(-m_{b}^{2},-m_{t}^{2},-m_{u}^{2},-m_{d}^{2}, Q^{2}, T^{2} ; M_{W}, m_{t}, 0, m_{u}\right) \\
& +C_{0}\left(-m_{u}^{2},-m_{d}^{2}, Q^{2} ; 0, m_{u}, M_{W}\right)-C_{0}\left(-m_{t}^{2},-m_{u}^{2}, T^{2} ; m_{t}, 0, m_{u}\right) .
\end{aligned}
$$

Let us emphasize that we have changed the ordering of mass arguments of $J_{W A}^{d}$ as compared to $D_{0}$. For $J_{W A}^{d}$ they are ordered into two pairs of heavy $(b, t)$ and light $(d, u)$ quarks such that the first mass in each pair corresponds to the fermion coupled to the photon, leading to the appearance of a contribution logarithmically singular in this mass. 
The great advantage of basic relations (11) is the following. The complex object $D_{0}$, containing an infrared divergence, is excluded in favor of an explicitly computed $J$ function and simple objects, $C_{0}$ functions, whose infrared divergences can be regularized by any method: by a photon mass, by dimensional regularization or by the width of an unstable particle. Examples of the latter $C_{0}$ functions, regularized by the width, may be found in Ref. [4].

We use the standard SANC definitions:

$$
Q^{2}=\left(p_{1}+p_{2}\right)^{2}, \quad T^{2}=\left(p_{2}+p_{3}\right)^{2}, \quad U^{2}=\left(p_{2}+p_{4}\right)^{2} .
$$

The paper is organized as follows.

Section 2 is devoted to the calculation of one of these functions $-J_{A W}^{d} \equiv J$. From here on we omit indices of $J$ since the list of arguments uniquely defines its type.

In Section 3 we present a similar calculation of the $J$ function for the process $u d \rightarrow t b$. The direct $A W$ and $W A$ functions are defined by:

$$
\begin{aligned}
& J_{A W}^{d}\left(Q^{2}, T^{2} ; m_{d}, m_{u}, m_{b}, m_{t}\right)= \\
& \quad\left(Q^{2}+M_{W}^{2}\right) D_{0}\left(-m_{d}^{2},-m_{u}^{2},-m_{t}^{2},-m_{b}^{2}, Q^{2}, T^{2} ; 0, m_{d}, M_{W}, m_{b}\right) \\
& \quad+C_{0}\left(-m_{t}^{2},-m_{b}^{2}, Q^{2} ; M_{W}, m_{b}, 0\right)-C_{0}\left(-m_{b}^{2},-m_{d}^{2}, T^{2} ; m_{b}, 0, m_{d}\right), \\
& J_{W A}^{d}\left(Q^{2}, T^{2} ; m_{u}, m_{d}, m_{t}, m_{b}\right)= \\
& \left(Q^{2}+M_{W}^{2}\right) D_{0}\left(-m_{d}^{2},-m_{u}^{2},-m_{t}^{2},-m_{b}^{2}, Q^{2}, T^{2} ; M_{W}, m_{u}, 0, m_{t}\right) \\
& +C_{0}\left(-m_{t}^{2},-m_{b}^{2}, Q^{2} ; 0, m_{t}, M_{W}\right)-C_{0}\left(-m_{u}^{2},-m_{t}^{2}, T^{2} ; m_{u}, 0, m_{t}\right) .
\end{aligned}
$$

Again, we limit ourselves to the presentation of function $J_{A W}^{d}$.

In Section 4 we briefly discuss the $J$ functions for the $t$ channel process $b u \rightarrow t d$.

For all processes we take the limit of vanishing light quark masses. The mass of the quark which is not coupled to the photon may be set equal to zero, while that for the quark coupled with the photon develops a mass singular logarithm. We keep logarithmic terms and neglect quark masses everywhere else. This approximation results in different expressions for $J$ functions for the three channels under consideration, and this is why the derivation must be presented for three channels separately.

Every Section ends by a numerical comparison with results obtained with the aid of the LoopTools package [5] for zero width and IR regularization by infinitesimal photon mass.

Section 5 contains a short introduction to the FORTRAN packages, which realize the calculation of "doubly subtracted" $J$ functions (see Sections 2-4 for their definition).

In Section 6 we present our conclusions.

\section{Calculation of the $J$ function for $t \rightarrow b u d$ decay}

\subsection{Representation in the form of a triple integral}

The basic definition of the function $J$ reads:

$$
i \pi^{2} J\left(Q^{2}, T^{2} ; m_{b}, m_{t}, m_{d}, m_{u}, M_{W}\right)=\mu^{4-n} \int d^{n} q \frac{-2 q \cdot p_{2}}{d_{0} d_{1} d_{2} d_{3}},
$$


where

$$
\begin{aligned}
& d_{0}=q^{2}, \quad d_{1}=\left(q+p_{1}\right)^{2}+m_{b}^{2} \\
& d_{2}=\left(q+p_{1}+p_{2}\right)^{2}+M_{W}^{2}, \quad d_{3}=\left(q-p_{4}\right)^{2}+m_{d}^{2} .
\end{aligned}
$$

By standard Feynman parametrization introducing variables $x, y, z$, as is shown in Fig. 1, one can pass to a $(3+n)$-tuple integral over $x, y, z$ and over the internal momentum $q$. In $n$-dimensional space we have:

$$
\int d^{n} q \frac{q_{\mu}}{\left(q^{2}-2 q p+m^{2}\right)^{\alpha}}=i \pi^{\frac{n}{2}} \frac{\Gamma\left(\alpha-\frac{n}{2}\right)}{\Gamma(\alpha)}\left(m^{2}-p^{2}\right)^{\frac{n}{2}-\alpha} p_{\mu} .
$$

In our case we have $m^{2}=L z, p=z k_{x y}, \alpha=n=4$, therefore Eq.(6) becomes:

$$
\int d^{4} q \frac{q_{\mu}\left(p_{4}\right)_{\mu}}{\left(q^{2}-2 z q k_{x y}+L z\right)^{4}}=i \pi^{2} \frac{\Gamma(2)}{\Gamma(4)}\left(L z-z^{2} k_{x y}^{2}\right)^{-2} z\left(k_{x y}\right)_{\mu}\left(p_{4}\right)_{\mu} .
$$

In terms of Feynman variables the denominator takes the form:

$$
D=d_{0}(1-z)+d_{1} z y(1-x)+d_{2} z x y+d_{3} z(1-y) .
$$

From expression (8) we derive:

$$
D=q^{2}-2 z q k_{x y}+L z-i \epsilon,
$$

where the variable $L$ and the vector $k_{x y}$ are:

$$
\begin{aligned}
L & =\left(Q^{2}+M_{W}^{2}\right) x y+\left(p_{1}^{2}+m_{b}^{2}\right)(1-x) y+\left(p_{4}^{2}+m_{d}^{2}\right)(1-y), \\
k_{x y} & =p_{4}(1-y)-p_{1} y(1-x)-\left(p_{1}+p_{2}\right) x y .
\end{aligned}
$$

Since $-i \epsilon$ is an infinitesimal addition, it is possible to replace it by $-i \epsilon z$ and redefine $L$ and $D$ :

$$
\begin{array}{r}
L=L-i \epsilon, \\
D=q^{2}-2 z q k_{x y}+L z .
\end{array}
$$

The triple integral over Feynman parameters may be expressed by the same Eqs. (13), (16)(17) as given in Ref. [3]:

$$
J=\int_{0}^{1} d x \int_{0}^{1} y d y N_{x y} \int_{0}^{1} d z \frac{z}{\left(L-z k_{x y}^{2}\right)^{2}}
$$


where we have neglected the light quark mass $m_{u}$ which does not lead to a mass singularity, and changed the notation of the other masses as follows:

$$
\begin{aligned}
m_{b} & \rightarrow m_{1}, \\
m_{t} & \rightarrow m_{2}, \\
M_{W} & \rightarrow m_{3}, \\
m_{d} & \rightarrow m_{4} .
\end{aligned}
$$

The ingredients entering Eq. (12) are

$$
\begin{aligned}
N_{x y} & =-2 k_{x y} p_{2}=-2 m_{2}^{2} x y+N_{y}, \\
N_{y} & =T^{2}(1-y)+y m_{2}^{2}+Q^{2}+m_{1}^{2},
\end{aligned}
$$

and

$$
\begin{aligned}
k_{x y}^{2} & =-m_{2}^{2} x^{2} y^{2}+N_{y} x y-T_{y}^{2}, \\
L & =P^{2} x y-i \epsilon \\
P^{2} & =Q^{2}+m_{3}^{2}, \\
T_{y}^{2} & =T^{2} y(1-y)+m_{1}^{2} y+m_{4}^{2}(1-y) .
\end{aligned}
$$

\section{$2.2 \quad$ Integration with respect to $z$}

The integration with respect to $z$ is straightforward:

$$
\int_{0}^{1} d z \frac{z}{\left(L-z k_{x y}^{2}\right)^{2}}=\frac{1}{\left(k_{x y}^{2}\right)^{2}}\left[\ln \left(L-k_{x y}^{2}\right)-\ln L+\frac{k_{x y}^{2}}{L-k_{x y}^{2}}\right] .
$$

\subsection{Integration with respect to $x$}

In Eqs. (12), (16) we perform, first of all, a change of variables:

$$
x y=x^{\prime}, \quad y d x=d x^{\prime},
$$

hence the ingredients become:

$$
\begin{aligned}
N_{x y} & =-2 m_{2}^{2} x+N_{y}, \\
k_{x y}^{2} & =-m_{2}^{2} x^{2}+N_{y} x-T_{y}^{2}, \\
L & =P^{2} x-i \epsilon .
\end{aligned}
$$

The key identity is

$$
N_{x y}=\frac{d}{d x} k_{x y}^{2} ;
$$


it allows the integration by parts in full analogy with section 2.3 of Ref. [3]:

$$
I(y)=\int_{0}^{y} d U V=\left.U V\right|_{\delta} ^{y}-\int_{\delta}^{y} d V U
$$

where we have introduced an infinitesimal parameter $\delta$, because both parts in Eq. (20) diverge separately.

Let

$$
L^{*}=L-k_{x y}^{2}
$$

be a quadratic trinomial in $x$ :

$$
L^{*}=A_{L} x^{2}+B_{L} x+C_{L}=A_{L}\left(x-x_{L_{1}}\right)\left(x-x_{L_{2}}\right),
$$

with coefficients

$$
\begin{aligned}
& A_{L}=m_{2}^{2} \\
& B_{L}=\left(T^{2}-m_{2}^{2}\right) y-T^{2}-m_{1}^{2}+m_{3}^{2}, \\
& C_{L}=T^{2} y(1-y)+m_{1}^{2} y+m_{4}^{2}(1-y)-i \epsilon
\end{aligned}
$$

and discriminant

$$
D_{L}=B_{L}^{2}-4 A_{L} C_{L}
$$

Next, we introduce the following notation: $\left.L^{*}\right|_{y}=L^{*}(x=y, y),\left.L^{*}\right|_{0}=L^{*}(x=0, y)$ and $k_{\left.x y\right|_{y}}^{2}=k_{x y}^{2}(x=y, y)$. The two binomials are

$$
\begin{aligned}
L_{\left.\right|_{y}}^{*} & =m_{3}^{2} y+m_{4}^{2}(1-y)-i \epsilon, \\
k_{\left.x y\right|_{y}}^{2} & =Q^{2} y-m_{4}^{2}(1-y) .
\end{aligned}
$$

For Eq. (20) one has

$$
d U=\frac{d k_{x y}^{2}}{\left(k_{x y}^{2}\right)^{2}}, \quad \frac{d V}{d x}=\frac{P^{2} k_{x y}^{2}}{L L^{*}}-\frac{k_{x y}^{2}}{\left(L^{*}\right)^{2}} \frac{d L^{*}}{d x},
$$

and the integral $I(y)$ becomes:

$$
I(y)=-\frac{1}{k_{x y \mid y}^{2}}\left[\ln \left(\left.L^{*}\right|_{y}\right)-\ln \left(L_{\mid y}\right)\right]-\frac{1}{T_{y}^{2}}\left[\ln \left(\left.L^{*}\right|_{0}\right)-\ln \left(L_{\left.\right|_{\delta}}\right)\right]+\int_{\delta}^{y} \frac{P^{2} d x}{L L^{*}} .
$$

After some more calculations we arrive at a one-dimensional integral over $y$, where the infinitesimal parameter $\delta$ cancels out. 


\section{$2.4 \quad$ Integration over $y$}

We proceed with the one-dimensional integral:

$$
J=\int_{0}^{1} d y I(y)
$$

with integrand

$$
I(y)=-\frac{1}{k_{x y \mid y}^{2}}\left[\ln \left(L_{\mid y}^{*}\right)-\ln \left(P^{2} y\right)\right]+\frac{1}{2 \sqrt{D_{T}}}\left(\frac{1}{y-y_{T_{1}}}-\frac{1}{y-y_{T_{2}}}\right) I_{p}(y),
$$

and

$$
\begin{aligned}
I_{p}(y)= & \ln \left(C_{L}\right)+\ln \left(L_{\mid y}^{*}\right)-2 \ln \left(P^{2} y\right) \\
& +\frac{B_{L}}{\sqrt{D_{L}}}\left[\ln \left(\frac{2 C_{L}+y\left(B_{L}+\sqrt{D_{L}}\right)}{C_{L}}\right)-\ln \left(\frac{2 C_{L}+y\left(B_{L}-\sqrt{D_{L}}\right)}{C_{L}}\right)\right] .
\end{aligned}
$$

Here $P$ gets redefined:

$$
P^{2}=Q^{2}+m_{3}^{2}-i \epsilon .
$$

The quadratic trinomial $C_{L}($ see $(23))$,

$$
\begin{aligned}
C_{L} & =a_{T} y^{2}+b_{T} y+c_{T} \\
a_{T} & =-T^{2} \\
b_{T} & =T^{2}+m_{1}^{2}-m_{4}^{2} \\
c_{T} & =m_{4}^{2}-i \epsilon \\
D_{T} & =b_{T}^{2}-4 a_{T} c_{T}
\end{aligned}
$$

has the roots:

$$
y_{T_{1,2}}=\frac{b_{T} \pm \sqrt{D_{T}}}{2 T^{2}} .
$$

The following steps of integration with respect to $y$ deviate from the presentation given in Ref. [3].

\subsubsection{Splitting into three parts}

Let us redistribute terms in Eqs. (29)-(30) into three parts:

$$
I(y)=I_{0}(y)+I_{1}(y)+I_{2}(y)
$$


with

$$
\begin{aligned}
I_{0}(y) & =\left[-\frac{1}{\left.k_{x y}^{2}\right|_{y}}+\frac{1}{\sqrt{D_{T}}}\left(\frac{1}{y-y_{T_{1}}}-\frac{1}{y-y_{T_{2}}}\right)\right]\left[\ln \left(1-\frac{y}{y_{l 0}}\right)-\ln \left(\frac{P^{2} y-i \epsilon}{m_{4}^{2}-i \epsilon}\right)\right], \\
I_{1}\left(y_{1}\right) & =\frac{1}{2 \sqrt{D_{T}}} \frac{1}{y_{1}}\left[N\left(y_{1}\right)+\frac{C_{1}}{\sqrt{D_{1}}}\left(M\left(y_{1}, \sqrt{D_{1}}\right)-M\left(y_{1},-\sqrt{D_{1}}\right)\right)\right] \\
I_{2}\left(y_{2}\right) & =-I_{1}\left(y_{1}\right) .
\end{aligned}
$$

For $I_{1}\left(y_{1}\right)$ and $I_{2}\left(y_{2}\right)$ we have changed the variables,

$$
y_{i}=y-y_{T_{i}}
$$

and used the notation

$$
\begin{aligned}
D_{i} & =D_{L \mid y \rightarrow y_{i}}=A_{i}^{2} y_{i}^{2}-2 B_{i} y_{i}+C_{i}^{2}, \\
A_{i} & \equiv A=T^{2}+m_{2}^{2} \\
B_{i} & =-A^{2} y_{T_{i}}+A\left(T^{2}+\Delta_{13}\right)+2 m_{2}^{2} \Delta_{34}, \\
C_{i} & =\left(T^{2}-m_{2}^{2}\right) y_{T i}-T^{2}-\Delta_{13} .
\end{aligned}
$$

Furthermore,

$$
\begin{aligned}
C\left(y_{1}\right) & =-y_{1}\left(T^{2} y_{1}+\sqrt{D_{T}}\right)=C\left(y_{2}\right)=-y_{2}\left(T^{2} y_{2}-\sqrt{D_{T}}\right) \\
N\left(y_{i}\right) & =\ln \left(\frac{C\left(y_{i}\right)}{m_{4}^{2}-i \epsilon}\right)-\ln \left(1-\frac{y}{y_{l 0}}\right) \\
M\left(y_{i}, \sqrt{D_{i}}\right) & =\ln \left(\frac{C\left(y_{i}\right)-m_{2}^{2}\left(y_{i}+y_{T_{i}}\right)^{2}+m_{4}^{2}-i \epsilon+\left(y_{i}+y_{T_{i}}\right)\left(\Delta_{34}+\sqrt{D_{i}}\right)}{C\left(y_{i}\right)}\right)
\end{aligned}
$$

and

$$
\begin{aligned}
y_{l 0} & =-\frac{\left(m_{4}^{2}-i \epsilon\right)}{\Delta_{34}}, \\
y_{d} & =\frac{m_{4}^{2}}{Q^{2}+m_{4}^{2}}, \\
k_{\left.x y\right|_{y}}^{2} & =\left(Q^{2}+m_{4}^{2}\right)\left(y-y_{d}\right), \\
\Delta_{i j} & =m_{i}^{2}-m_{j}^{2} .
\end{aligned}
$$

These expression are still valid for all $J^{\prime} s$ and are exact in $m_{4}$. The main idea of this splitting arises from the observation that the $m_{4}$ singularities are completely confined to $I_{0}$, hence when calculating $I_{1}$ and $I_{2}$ one may take the limit $m_{4} \rightarrow 0$ before taking the integrals. 


\subsubsection{Part $J_{0}$}

The integral for $J_{0}$ is straightforward, and we limit ourselves to presenting the answer in the limit $m_{4} \rightarrow 0$ :

$$
\begin{aligned}
J_{0}= & \int_{0}^{1} I_{0}(y) d y=\frac{1}{Q^{2}}\left[\ln \left(\frac{P^{2}}{m_{3}^{2}}\right) \ln \left(-\frac{Q^{2}}{m_{4}^{2}}\right)+\operatorname{Li}_{2}\left(\frac{P^{2}}{m_{3}^{2}}\right)-\zeta(2)\right] \\
& +\frac{1}{\sqrt{D_{T}}}\left\{-\ln \left(\frac{P^{2}}{m_{3}^{2}}\right) \ln \left(\frac{m_{3}^{2}}{m_{4}^{2}}\right)-\ln \left(\frac{P^{2}}{m_{3}^{2}}\right) \ln \left(R_{13}\right)\right. \\
& \left.+\ln \left(\frac{P^{2}}{m_{3}^{2}}\right) \ln \left(1-\frac{T^{2}}{T^{2}+m_{1}^{2}-i \epsilon}\right)-\operatorname{Li}_{2}\left(1-R_{13}\right)+\zeta(2)\right\},
\end{aligned}
$$

where

$$
R_{1 i}=\frac{T^{2}+m_{1}^{2}-i \epsilon}{m_{i}^{2}}, \quad i=1,3
$$

and $\operatorname{Li}_{2}(x)$ is the dilogarithm function defined by $\operatorname{Li}_{2}(x)=-\int_{0}^{1} \frac{\ln |1-x t|}{t} d t$.

\subsubsection{Part $J_{1}$}

For $J_{1}$ we directly take the limit $m_{4}=0$ in which it becomes

$$
J_{1}=\int_{0}^{1} I_{1}(y) d y=\int_{0}^{1} d y \frac{1}{2 \sqrt{D_{T}}} \frac{1}{y}\left[\ln \left(\frac{T^{2}(1-y)+m_{1}^{2}-i \epsilon}{m_{3}^{2}-i \epsilon}\right)+\frac{C_{1}}{\sqrt{D_{1}}} \ln (R(y))\right],
$$

with the ratio

$$
R(y)=\frac{T_{13}^{2}-A_{1} y+\sqrt{D_{1}}}{T_{13}^{2}-A_{1} y-\sqrt{D_{1}}}
$$

where, since we neglect $m_{4}$,

$$
\begin{aligned}
D_{1} & \equiv D_{1}(y)=A_{1}^{2} y^{2}-2 B_{1} y+C_{1}^{2}, \\
A_{1} & \equiv A=T^{2}+m_{2}^{2}, \\
B_{1} & =A T_{13}^{2}-2 T^{2}\left(m_{3}^{2}-i \epsilon\right), \\
C_{1} & =-\left(T^{2}+m_{1}^{2}-m_{3}^{2}\right), \\
T_{13}^{2} & =T^{2}+m_{1}^{2}+m_{3}^{2}-2 i \epsilon .
\end{aligned}
$$

Then we use the substitution:

$$
\sqrt{D_{1}(y)}=C_{1}+\frac{E x-B_{1}}{C_{1}} y
$$

where

$$
E=C_{1} \sqrt{D_{1}(y)_{\mid y=1}}-C_{1}^{2}+B_{1} .
$$


From Eq. (45) we have:

$$
\begin{aligned}
y & =2 \frac{C_{1}^{2} E x}{d(x)}, \\
\sqrt{D_{1}(y)} & =\frac{C_{1} n(x)}{d(x)}, \\
\frac{d y}{d x} & =2 \frac{C_{1}^{2} E n(x)}{d^{2}(x)}, \\
\frac{d y}{y \sqrt{D_{1}(y)}} & =\frac{d x}{C_{1} x}
\end{aligned}
$$

where

$$
\begin{aligned}
& d(x)=A^{2} C_{1}^{2}-\left(E x-B_{1}\right)^{2}, \\
& n(x)=A^{2} C_{1}^{2}-B_{1}^{2}+E^{2} x^{2} .
\end{aligned}
$$

In order to take the integral (42) we need to know the limits of variation of $x$. Let

$$
\delta \leq y \leq 1
$$

then

$$
x_{\left.\right|_{\delta}} \equiv \frac{A^{2} C_{1}^{2}-B_{1}^{2}}{2 C_{1}^{2} E} \delta \leq x \leq 1
$$

Next, replacing the variable $y$ in the ratio (43) by $x$, we find

$$
R(x)=\frac{\left(T_{13}^{2}+C_{1}\right)\left[1-E x /\left(B_{1}+A C_{1}\right)\right]\left[1-E x\left(T_{13}^{2}-C_{1}\right) /\left(T_{13}^{2}+C_{1}\right) /\left(B_{1}-A C_{1}\right)\right]}{\left(T_{13}^{2}-C_{1}\right)\left[1-E x /\left(B_{1}-A C_{1}\right)\right]\left[1-E x\left(T_{13}^{2}+C_{1}\right) /\left(T_{13}^{2}-C_{1}\right) /\left(B_{1}+A C_{1}\right)\right]} .
$$

Taking account of all above equations, we rewrite the integral $J_{1}$ of Eq. (42) using both variables $x$ or $y$ where convenient:

$$
\begin{aligned}
J_{1}= & \frac{1}{2 \sqrt{D_{T}}}\left\{\ln \left(\frac{T^{2}+m_{1}^{2}-i \epsilon}{m_{3}^{2}-i \epsilon}\right) \int_{\delta}^{1} \frac{d y}{y}+\int_{0}^{1} \frac{d y}{y} \ln \left(1-\frac{T^{2} y}{T^{2}+m_{1}^{2}-i \epsilon}\right)\right. \\
& +\ln \left(\frac{T_{13}^{2}+C_{1}}{T_{13}^{2}-C_{1}}\right) \int_{x_{\mid \delta}}^{1} \frac{d x}{x} \\
& +\frac{T_{13}^{2}}{C_{1}} \int_{0}^{1} \frac{d x}{x}\left[-\ln \left(1-\frac{E x}{\left(B_{1}+A C_{1}\right)}\right)-\ln \left(1-\frac{E\left(T_{13}^{2}-C_{1}\right) x}{\left(T_{13}^{2}+C_{1}\right)\left(B_{1}-A C_{1}\right)}\right)\right. \\
& \left.\left.\quad+\ln \left(1-\frac{E x}{\left(B_{1}-A C_{1}\right)}\right)+\ln \left(1-\frac{E\left(T_{13}^{2}+C_{1}\right) x}{\left(T_{13}^{2}-C_{1}\right)\left(B_{1}+A C_{1}\right)}\right)\right]\right\} .
\end{aligned}
$$


Finally we get

$$
\begin{aligned}
J_{1}= & \frac{1}{2 \sqrt{D_{T}}}\left\{\ln \left(\frac{T^{2}+m_{1}^{2}}{m_{3}^{2}}\right) \ln \left(\frac{A^{2} C_{1}^{2}-B_{1}^{2}}{2 C_{1}^{2} E}\right)\right. \\
& -\operatorname{Li}_{2}\left(\frac{T^{2}}{T^{2}+m_{1}^{2}}\right)-\operatorname{Li}_{2}\left(\frac{E}{B_{1}+A C_{1}}\right)-\operatorname{Li}_{2}\left(\frac{E\left(T_{13}^{2}-C_{1}\right)}{\left(T_{13}^{2}+C_{1}\right)\left(B_{1}-A C_{1}\right)}\right) \\
& \left.+\operatorname{Li}_{2}\left(\frac{E}{B_{1}-A C_{1}}\right)+\operatorname{Li}_{2}\left(\frac{E\left(T_{13}^{2}+C_{1}\right)}{\left(T_{13}^{2}-C_{1}\right)\left(B_{1}+A C_{1}\right)}\right)\right\} .
\end{aligned}
$$

\subsubsection{Part $J_{2}$}

For $I_{2}\left(y_{2}\right)$ of $(35)$ we set

$$
y_{2}=y-y_{T_{2}}
$$

where in the limit $m_{4} \rightarrow 0$

$$
y_{T 2}=1+\frac{m_{1}^{2}-i \epsilon}{T^{2}} .
$$

- Transition from variable $y_{2}$ to variable $t_{2}$

At this step we make the transition from the variable $y_{2}$ to $t_{2}$ by

$$
\sqrt{D_{2}}=\sqrt{A^{2} y_{2}^{2}-2 B_{2} y_{2}+C_{2}^{2}}=\left|C_{2}\right|+y_{2} t_{2} .
$$

The presence of $\left|C_{2}\right|$ leads to the appearance of two branches, $\left|C_{2}\right|= \pm C_{2}>0$, in the final answer for $J_{2}$. For our definitions see Eqs. (38).

As a consequence of (56) we get

$$
y_{2}=2 \frac{B_{2}+C_{2} t_{2}}{A_{2}^{2}-t_{2}^{2}}, \quad \sqrt{D_{2}}=\frac{N_{2}}{A_{2}^{2}-t_{2}^{2}},
$$

with

$$
N_{2}=A_{2}^{2} C_{2}+2 B_{2} t_{2}+C_{2} t_{2}^{2}
$$

The Jacobian of the transition is

$$
\frac{d y_{2}}{d t_{2}}=2 \frac{N_{2}}{\left(A^{2}-t_{2}^{2}\right)^{2}}
$$

We also need

$$
\frac{d y_{2}}{y_{2}}=\frac{N_{2} d t_{2}}{\left(B_{2}+C_{2} t_{2}\right)\left(A^{2}-t_{2}^{2}\right)}=\left(\frac{C_{2}}{B_{2}+C_{2} t_{2}}+\frac{1}{A-t_{2}}-\frac{1}{A+t_{2}}\right) d t_{2},
$$

and

$$
\frac{d y_{2}}{y_{2} \sqrt{D_{2}}}=\frac{d t_{2}}{B_{2}+C_{2} t_{2}}
$$


The limits of integration are

$$
t_{2}^{\max }=\frac{s_{D}^{\max }-C_{2}^{ \pm}}{1-y_{T 2}}, \quad t_{2}^{\min }=\frac{s_{D}^{\min }-C_{2}^{ \pm}}{-y_{T 2}}
$$

The remaining variables read

$$
\begin{aligned}
& s_{D}^{\max }=\sqrt{\left(m_{2}^{2}+\Delta_{13}\right)^{2}-4 m_{2}^{2}\left(m_{1}^{2}-i \epsilon\right)}, \\
& s_{D}^{\min }=\sqrt{\left(T^{2}+\Delta_{13}\right)^{2}-4 m_{2}^{2}\left(m_{4}^{2}-i \epsilon\right)} .
\end{aligned}
$$

- Replacement of variable from $t_{2}$ to $y^{\prime} \equiv y$.

The next replacement reads:

$$
t_{2}=D_{t 2} y+t_{2}^{\min }
$$

where

$$
D_{t 2}=t_{2}^{\max }-t_{2}^{\min }
$$

The Jacobian of this transition is

$$
\frac{d t_{2}}{B_{2}+C_{2} t_{2}}=\frac{d y}{C_{2}\left(y-y_{d 2}\right)} .
$$

The root $y_{d 2}$ is labelled by a second index \pm , depending on the $\operatorname{sign}$ of $C_{2}^{ \pm}$.

$$
y_{d 2}^{ \pm}=-\frac{B_{2}+C_{2}^{ \pm} t_{2}^{\min }}{C_{2}^{ \pm} D_{t 2}} .
$$

For $I_{2}\left(y_{2}\right)$ we get from Eq. (35)

$$
I_{2}\left(y_{2}\right)=-\frac{1}{2 \sqrt{D_{T}} y_{2}}\left\{N\left(y_{2}\right)+\frac{C_{2}^{\mp}}{\sqrt{D_{2}}}\left[M\left(y_{2}, \sqrt{D_{2}}\right)-M\left(y_{2},-\sqrt{D_{2}}\right)\right]\right\},
$$

and

$$
C_{2}^{\mp}= \pm C_{2} .
$$

The second term in curly brackets of (68), after replacement of variables (56), reads:

$$
\begin{aligned}
& \frac{C_{2}^{+}}{y_{2} \sqrt{D_{2}}}\left[M\left(y_{2}, \sqrt{D_{2}}\right)-M\left(y_{2},-\sqrt{D_{2}}\right)\right]=\frac{C_{2}^{+}}{y_{2} \sqrt{D_{2}}}\left[\ln \left(\frac{n_{2}^{++}}{d_{2}^{+}}\right)-\ln \left(\frac{n_{2}^{+-}}{d_{2}^{+}}\right)\right], \\
& \frac{C_{2}^{-}}{y_{2} \sqrt{D_{2}}}\left[M\left(y_{2}, \sqrt{D_{2}}\right)-M\left(y_{2},-\sqrt{D_{2}}\right)\right]=\frac{C_{2}^{-}}{y_{2} \sqrt{D_{2}}}\left[\ln \left(\frac{n_{2}^{-+}}{d_{2}^{-}}\right)-\ln \left(\frac{n_{2}^{--}}{d_{2}^{-}}\right)\right],
\end{aligned}
$$




$$
\begin{aligned}
d_{2}^{ \pm} & =-2 T^{2}\left(B_{2}+C_{2}^{ \pm} t_{2}\right), \\
n_{2}^{-+/+-} & = \pm 2\left(B_{2}+C_{2}^{\mp} t_{2}\right)\left(t_{2} \mp A\right), \\
n_{2}^{++/--} & = \pm 2\left(B_{2} \mp A C_{2}^{ \pm}\right)\left(t_{2} \mp A\right) .
\end{aligned}
$$

- Solution $C_{2}^{-}$

$$
I_{2}\left(y_{2}\right)=-\frac{1}{2 \sqrt{D_{T}} y_{2}}\left\{N\left(y_{2}\right)+\frac{C_{2}^{-}}{\sqrt{D_{2}}}\left[\ln \left(\frac{n_{2}^{-+}}{d_{2}^{-}}\right)-\ln \left(\frac{n_{2}^{--}}{d_{2}^{-}}\right)\right]\right\},
$$

with

$$
C_{2}=C_{2}^{-}=\left(1+\frac{m_{1}^{2}-i \epsilon}{T^{2}}\right) m_{2}^{2}-\left(m_{3}^{2}-i \epsilon\right) .
$$

For $N$ and part of $M$ we derive:

$$
\begin{aligned}
N\left(y_{2}\right) & =\ln \left(\frac{-T^{2}}{m_{3}^{2}-i \epsilon}\right)+\ln \left(y_{2}\right), \\
\ln \left(\frac{n_{2}^{-+}}{d_{2}^{-}}\right) & =\ln \left(\frac{y_{l-} D_{t 2}}{T^{2}}\right)+\ln \left(1-\frac{y}{y_{l-}}\right), \\
\ln \left(\frac{n_{2}^{--}}{d_{2}^{-}}\right) & =\ln \left(\frac{y_{l+} D_{t 2}\left(y_{l-}-y_{d 2}\right)}{T^{2} y_{d 2}}\right)+\ln \left(1-\frac{y}{y_{l+}}\right)-\ln \left(1-\frac{y}{y_{d 2}}\right), \\
\ln \left(y_{2}\right) & =\ln \left(\frac{2 C_{2} y_{d 2}}{y_{l-} y_{l+} D_{t 2}}\right)+\ln \left(1-\frac{y}{y_{d 2}}\right)-\ln \left(1-\frac{y}{y_{l-}}\right)-\ln \left(1-\frac{y}{y_{l+}}\right),
\end{aligned}
$$

where

$$
\begin{aligned}
& y_{d 2}=-\frac{B_{2}+t_{2}^{\text {min }} C_{2}}{D_{t 2} C_{2}}, \\
& y_{l-}=+\frac{A-t_{2}^{\text {min }}}{D_{t 2}}, \\
& y_{l+}=-\frac{A+t_{2}^{\text {min }}}{D_{t 2}} .
\end{aligned}
$$

Putting the variable substitutions and Eqs. (74) into Eq. (72), one gets:

$$
J_{2}=-\frac{1}{2 \sqrt{D_{T}}} \ln \left(\frac{-T^{2}}{m_{3}^{2}-i \epsilon}\right) \ln \left(\frac{1-y_{T 2}}{-y_{T 2}}\right)+J_{2}^{\prime},
$$

with

$$
\begin{aligned}
J_{2}^{\prime}= & \frac{1}{2\left(T^{2}+m_{1}^{2}\right)}\left\{-\ln \left(\frac{2 C_{2} y_{d 2}}{y_{l-} y_{l+} D_{t 2}}\right)\left[l\left(y_{l+}\right)+\left(y_{l-}\right)\right]+\frac{1}{2} l^{2}\left(y_{l+}\right)+\frac{1}{2} l^{2}\left(y_{l-}\right)\right. \\
& +\left[\ln \left(\frac{2 C_{2} y_{d 2}}{y_{l-} y_{l+} D_{t 2}}\right)-\ln \left(\frac{y_{l-} D_{t 2}}{T^{2}}\right)+\ln \left(\frac{y_{l+} D_{t 2}\left(y_{l-}-y_{d 2}\right)}{T^{2} y_{d 2}}\right)\right] l\left(y_{d 2}\right) \\
& \left.+\mathcal{M}\left(y_{l-}, y_{l+}\right)-\mathcal{M}\left(y_{d 2}, y_{l+}\right)+\mathcal{M}\left(y_{l+}, y_{l-}\right)-\mathcal{M}\left(y_{d 2}, y_{l-}\right)-2 \mathcal{M}\left(y_{l-}, y_{d 2}\right)\right\}
\end{aligned}
$$


and

$$
l(y)=\ln \left(1-\frac{1}{y}\right)
$$

and the "master integral":

$$
\mathcal{M}\left(y_{l}, y_{d}\right)=\int_{0}^{1} \frac{d y}{\left(y-y_{d}\right)} \ln \left(1-\frac{y}{y_{l}}\right)=\ln \left(1-\frac{y_{d}}{y_{l}}\right) l\left(y_{d}\right)-\operatorname{Li}_{2}\left(\frac{1-y_{d}}{y_{l}-y_{d}}\right)+\operatorname{Li}_{2}\left(\frac{-y_{d}}{y_{l}-y_{d}}\right) .
$$

- Solution $C_{2}^{+}$

$$
I_{2}\left(y_{2}\right)=-\frac{1}{2 \sqrt{D_{T}} y_{2}}\left\{N\left(y_{2}\right)+\frac{C_{2}^{+}}{\sqrt{D_{2}}}\left[\ln \left(\frac{n_{2}^{++}}{d_{2}^{+}}\right)-\ln \left(\frac{n_{2}^{+-}}{d_{2}^{+}}\right)\right]\right\},
$$

with

$$
C_{2}=C_{2}^{+}=-\left(1+\frac{m_{1}^{2}-i \epsilon}{T^{2}}\right) m_{2}^{2}+\left(m_{3}^{2}-i \epsilon\right) .
$$

The derivation is similar and we limit ourselves to presenting the answer for $J_{2}^{\prime}$ :

$$
\begin{aligned}
J_{2}^{\prime}= & \frac{1}{2\left(T^{2}+m_{1}^{2}\right)}\left\{-\ln \left(\frac{2 C_{2} y_{d 2}}{y_{l-} y_{l+} D_{t 2}}\right)\left[l\left(y_{l+}\right)+\left(y_{l-}\right)\right]+\frac{1}{2} l^{2}\left(y_{l+}\right)+\frac{1}{2} l^{2}\left(y_{l-}\right)\right. \\
& +\left[\ln \left(\frac{2 C_{2} y_{d 2}}{y_{l-} y_{l+} D_{t 2}}\right)-\ln \left(-\frac{y_{l+} D_{t 2}}{T^{2}}\right)+\ln \left(\frac{y_{l-} D_{t 2}\left(y_{d 2}-y_{l+}\right)}{T^{2} y_{d 2}}\right)\right] l\left(y_{d 2}\right) \\
& \left.+\mathcal{M}\left(y_{l-}, y_{l+}\right)-\mathcal{M}\left(y_{d 2}, y_{l+}\right)+\mathcal{M}\left(y_{l+}, y_{l-}\right)-\mathcal{M}\left(y_{d 2}, y_{l-}\right)-2 \mathcal{M}\left(y_{l+}, y_{d 2}\right)\right\} .
\end{aligned}
$$

\subsection{Definitions of functions $J_{\text {sub }}^{t b u d}$}

The mass singularities in arguments of the logarithms may be compensated by combining $J$

with one more $C_{0}$ function:

$$
\begin{aligned}
J_{\text {sub }}^{\text {tbud }}\left(Q^{2}, T^{2} ; m_{b}, m_{t}, M_{W}\right)= & J\left(Q^{2}, T^{2} ; m_{b}, m_{t}, m_{d}, m_{u}, M_{W}\right) \\
& -\left(1+\frac{Q^{2}}{m_{b}^{2}+T^{2}}\right) C_{0}\left(-m_{u}^{2},-m_{d}^{2}, Q^{2} ; M_{W}, m_{d}, 0\right), \\
J_{\text {sub }}^{\text {tbud }}\left(Q^{2}, T^{2} ; m_{t}, m_{b}, M_{W}\right)= & J\left(Q^{2}, T^{2} ; m_{t}, m_{b}, m_{u}, m_{d}, M_{W}\right) \\
& -\left(1+\frac{Q^{2}}{m_{t}^{2}+T^{2}}\right) C_{0}\left(-m_{d}^{2},-m_{u}^{2}, Q^{2} ; M_{W}, m_{u}, 0\right) .
\end{aligned}
$$

where $T^{2}=U^{2}$ for cross functions. The two mass-singular $C_{0}$ functions appearing in Eqs. (83) and (84) cancel in the total expression for the EW correction which proves the absence in it of logarithmic mass singularities (not KLN theorem!). 


\subsubsection{Calculation of the subtracted function $J_{\text {sub }}^{t b u d}$}

The first function $J_{\text {sub }}^{\text {tbud }}$ is given by equation (83), where we may neglect $m_{u}$ which does not give rise to a mass singularity:

$$
J_{\text {sub }}^{\text {tbud }}=J-\left(1+\frac{Q^{2}}{T^{2}+m_{1}^{2}}\right) C_{0}\left(0, m_{4}^{2}, Q^{2} ; m_{3}, m_{4}, 0\right) .
$$

For $C_{0}$ we have:

$$
C_{0}\left(0, m_{4}^{2}, Q^{2} ; m_{3}, m_{4}, 0\right)=\frac{1}{Q^{2}}\left[\ln \left(-\frac{Q^{2}}{m_{4}^{2}}\right) \ln \left(R_{3}\right)+\operatorname{Li}_{2}\left(R_{3}\right)-\zeta(2)\right],
$$

with

$$
R_{3}=\frac{Q^{2}+m_{3}^{2}-i \epsilon}{m_{3}^{2}}
$$

For $J_{\text {sub }}^{\text {tbud }}$ one derives an expression for $J_{0}$ that is free of the $m_{4}$ mass singularity; $J_{1,2}$ remain unchanged:

$$
\begin{gathered}
J_{\text {sub }}^{t \text { bud }}=J_{\text {sub }, 0}^{t b u d}+J_{1}+J_{2}, \\
J_{\text {sub }, 0}^{t b u d}=-\frac{1}{T^{2}+m_{1}^{2}}\left\{\ln \left(R_{3}\right)\left[\ln \left(-\frac{Q^{2}}{m_{3}^{2}}\right)-\ln \left(R_{13}\right)-\ln \left(R_{11}\right)\right]+\operatorname{Li}_{2}\left(R_{3}\right)-\operatorname{Li}_{2}\left(1-R_{13}\right)\right\} .
\end{gathered}
$$

\subsection{Definitions of the function $J_{\text {subsub }}^{t b u d}$}

If we want to neglect the $b$ quark mass, $m_{b}$, we must perform a second subtraction of the mass singular $C_{0}$ function $C_{0}\left(-m_{t}^{2},-m_{b}^{2}, Q^{2}, M_{W}, m_{b}, 0\right)$ that appears in the limit $m_{b} \rightarrow 0$.

Note that only one of $J_{\text {sub }}^{\text {tbud }}$ contains an $m_{b}$ mass singularity.

$$
\begin{aligned}
J_{\text {subsub }, 1}^{\text {tbud }}\left(Q^{2}, T^{2} ; m_{b}, m_{t}, M_{W}\right)= & J_{\text {sub }}^{\text {tbud }}\left(Q^{2}, T^{2} ; m_{b}, m_{t}, M_{W}\right) \\
& -\frac{Q^{2}+m_{t}^{2}}{T^{2}} C_{0}\left(-m_{t}^{2},-m_{b}^{2}, Q^{2} ; M_{W}, m_{b}, 0\right) .
\end{aligned}
$$

Again, the $m_{b}$ mass singular $C_{0}$ function $C_{0}\left(-m_{t}^{2},-m_{b}^{2}, Q^{2} ; M_{W}, m_{b}, 0\right)$ cancels in the total EW correction.

Since we do not want to consider the limit $m_{t}=0$, we simply rename the second function:

$$
J_{\text {subsub }, 2}^{\text {tbud }}\left(Q^{2}, T^{2} ; m_{t}, m_{b}, M_{W}\right)=J_{\text {sub }}^{\text {tbud }}\left(Q^{2}, T^{2} ; m_{t}, m_{b}, M_{W}\right),
$$

assuming $m_{b}=0$ for this, non-singular case.

\subsubsection{The first function $J_{\text {sub }}^{\text {tbud }}$ in the limit $m_{b} \rightarrow 0$}

Here we simply present the limits of $J_{0,2}$ which develop $m_{1}$ mass singular logarithms; for $J_{1}$ it is sufficient to set $m_{1}=0$ in Eq. (53).

$$
\begin{aligned}
J_{0}= & \frac{1}{T^{2}}\left\{\ln \left(\frac{P}{m_{3}^{2}}\right)\left[2 \ln \left(\frac{T^{2}-i \epsilon}{m_{3}^{2}}\right)+\ln \left(\frac{m_{3}^{2}}{m_{1}^{2}}\right)\right]-\frac{1}{2} \ln \left(-\frac{T^{2}}{m_{3}^{2}}\right) \ln \left(\frac{T^{2}-i \epsilon}{m_{1}^{2}}\right)\right. \\
& \left.-\ln \left(\frac{P}{m_{3}^{2}}\right) \ln \left(-\frac{Q^{2}}{m_{3}^{2}-i \epsilon}\right)-\operatorname{Li}_{2}\left(R_{3}\right)+\operatorname{Li}_{2}\left(-\frac{T^{2}-m_{3}^{2}-i \epsilon}{m_{3}^{2}}\right)\right\} .
\end{aligned}
$$


Here $P$ is given by Eq. (31). For the $J_{2}$ part one finds:

$$
\begin{aligned}
J_{2}= & \frac{1}{T^{2}}\left[\ln \left(\frac{\Delta_{23}+i \epsilon}{T^{2}}\right)+\frac{1}{2} \ln \left(-\frac{T^{2}}{m_{3}^{2}}\right)\right] \ln \left(\frac{m_{1}^{2}}{m_{3}^{2}}\right) \\
& +\frac{1}{2 T^{2}}\left\{\frac{1}{2} l^{2}\left(y_{l+}\right)+i \pi l\left(y_{l+}\right)+\frac{1}{2} l^{2}\left(y_{l-}\right)+i \pi l\left(y_{l-}\right)\right. \\
& +\left[2 \ln \left(\frac{\Delta_{23}+i \epsilon}{T^{2}}\right)+\ln \left(-\frac{T^{2}}{m_{3}^{2}}\right)\right] \ln \left(-\frac{m_{3}^{2} m_{2}^{2}}{\tilde{\Delta}_{23}^{2}}\right) \\
& \left.+\mathcal{M}\left(y_{l-}, y_{l+}\right)-\mathcal{M}\left(1, y_{l+}\right)+\mathcal{M}\left(y_{l+}, y_{l-}\right)-\mathcal{M}\left(1, y_{l-}\right)-2 \operatorname{Li}_{2}\left(\frac{1}{1-y_{l-}}\right)\right\}
\end{aligned}
$$

where

$$
y_{l-}=\frac{T^{2}}{\left(T^{2}-i \epsilon\right)} \frac{\tilde{\Delta}_{23}}{\left(\tilde{\Delta}_{23}+T^{2}\right)}, \quad y_{l+}=-\frac{\tilde{\Delta}_{23}}{m_{3}^{2}},
$$

and

$$
\tilde{\Delta}_{23}=m_{2}^{2}-\left(m_{3}^{2}-i \epsilon\right)
$$

Summing Eqs. (92) and (93) with Eq. (53) in the limit of $m_{1}=0$ we get:

$$
J_{\text {sub }}^{\text {tbud }}=J_{0}+J_{1}+J_{2}
$$

\subsubsection{The function $J_{\text {subsub,1 }}^{\text {tbud }}$}

The $C_{0}$ function needed in Eq. (90) with explicitly separated out $m_{2}$ mass singular logarithm looks as follows:

$$
\begin{aligned}
& C_{0}\left(p_{1}^{2}, p_{2}^{2}, Q^{2} ; m_{1}^{2}, m_{2}^{2}, 0\right)=\frac{1}{Q^{2}-p_{1}^{2}}\left\{\ln \left(\frac{m_{1}^{2}}{m_{2}^{2}}\right) \ln \left(\frac{P^{2}}{p_{1}^{2}+m_{1}^{2}-i \epsilon}\right)\right. \\
& +\left[\ln \left(-\frac{\left(Q^{2}-p_{1}^{2}\right)^{2}}{m_{1}^{2} Q^{2}}\right)-2 i \pi\right] \ln \left(\frac{P^{2}}{m_{1}^{2}}\right)-\left[\ln \left(-\frac{\left(Q^{2}-p_{1}^{2}\right)^{2}}{m_{1}^{2} p_{1}^{2}}\right)-2 i \pi\right] \ln \left(\frac{p_{1}^{2}+m_{1}^{2}-i \epsilon}{m_{1}^{2}}\right) \\
& \left.-\mathrm{Li}_{2}\left(\frac{P^{2}}{m_{1}^{2}}\right)+\operatorname{Li}_{2}\left(\frac{p_{1}^{2}+m_{1}^{2}-i \epsilon}{m_{1}^{2}}\right)+\operatorname{Li}_{2}\left(\frac{P^{2}}{p_{1}^{2}+m_{1}^{2}}\right)+\operatorname{Li}_{2}\left(\frac{\left(P^{2}\right)^{*}}{p_{1}^{2}+m_{1}^{2}}\right)-2 \zeta(2)\right\} .
\end{aligned}
$$

Here $P^{2}=Q^{2}+m_{1}^{2}-i \epsilon$ and $p_{2}^{2}=-m_{2}^{2}$.

Let us emphasize that in this section $C_{0}$ has its own list of dummy arguments!

In order to derive $J_{\text {subsub, }}^{\text {tbud }}$ it is sufficient to redefine $J_{0}$ of (92) into $J_{0}^{\prime}$ by summing it with the first row of $J_{2}$ of (93) and the $C_{0}$ function of (97) with the coefficient of Eq. (83), i.e. collect together all $m_{b}$ mass singular terms; that is:

$$
J_{\text {subsub }, 1}^{\text {tbud }}=J_{0}^{\prime}+J_{1}+J_{2}^{\prime}
$$

where $J_{1}$ remains unchanged, given as before by the limit of Eq. (53) at $m_{1}=0$, and $J_{2}^{\prime}$ denotes the rest of $J_{2}$ without its first row. 
For $J_{0}^{\prime}$ we get:

$$
\begin{aligned}
J_{0}^{\prime}= & \frac{1}{T^{2}}\left\{\ln \left(-\frac{T^{2}-i \epsilon}{Q^{2}+m_{2}^{2}}\right) \ln \left(\frac{P^{2}}{m_{3}^{2}}\right)+\left[\ln \left(-\frac{Q^{2}+m_{2}^{2}}{m_{3}^{2}-i \epsilon}\right)+\ln \left(\frac{m_{3}^{2}}{m_{2}^{2}}\right)\right] \ln \left(-\frac{\tilde{\Delta}_{23}}{m_{3}^{2}}\right)\right. \\
& -\frac{1}{2} \ln \left(-\frac{T^{2}-i \epsilon}{m_{3}^{2}}\right) \ln \left(\frac{T^{2}-i \epsilon}{m_{3}^{2}}\right)+\operatorname{Li}_{2}\left(-\frac{T^{2}-m_{3}^{2}-i \epsilon}{m_{3}^{2}}\right) \\
& \left.-\operatorname{Li}_{2}\left(-\frac{\tilde{\Delta}_{23}}{m_{3}^{2}}\right)-\operatorname{Li}_{2}\left(-\frac{P^{2}}{\Delta_{23}}\right)-\operatorname{Li}_{2}\left(-\frac{\left(P^{2}\right)^{*}}{\Delta_{23}}\right)+2 \zeta(2)\right\}
\end{aligned}
$$

with $\tilde{\Delta}_{23}$ defined by Eq. (95).

In Table1 we give a comparison of real and imaginary parts of the function $J_{\text {subsub, } 1}^{t b u d}$ defined by Eq. (90) and related ones, computed with the aid of the LoopTools package [5], vs numbers derived exactly from Eq. (98) with $J_{0}^{\prime}$ given by Eq. (99) and $J_{1}$ and $J_{2}^{\prime}$ by previous equations as explained just below Eq. (98), i.e. results of this paper.

The numbers are given for four values of $s$ (the first two values near the kinematical edges and the latter two in the $\pm 1 \mathrm{GeV}$ vicinity of the $W$ resonance) and for three values of $\cos \theta$ at $m_{\gamma}=10^{-40}, m_{1}=10^{-7}, m_{2}=174.3, m_{3}=80.403, m_{4}=5 \cdot 10^{-7}$ (all masses are given in $\mathrm{GeV}$ ); first lines — LoopTools, second lines — this paper.

Table 1: Comparison of $J_{\text {subsub }, 1}^{\text {tbud }}$

\begin{tabular}{||c|c|c||}
\hline \hline $\cos \theta$ & $\sqrt{s}=1 \mathrm{GeV}$ & $\sqrt{s}=173.2 \mathrm{GeV}$ \\
\hline-0.999 & $-0.374523885975 \mathrm{E}-7,0.483905777792 \mathrm{E}-7$ & $0.220728294741 \mathrm{E}-1,-0.404470353912 \mathrm{E}-1$ \\
& $-0.374523675918 \mathrm{E}-7,0.483905777797 \mathrm{E}-7$ & $0.220728294741 \mathrm{E}-1,-0.404470353912 \mathrm{E}-1$ \\
\hline 0 & $-0.103142713149 \mathrm{E}-3,0.143516783301 \mathrm{E}-3$ & $0.490255707173 \mathrm{E}-1,-0.933810555325 \mathrm{E}-1$ \\
& $-0.103142713128 \mathrm{E}-3,0.143516783301 \mathrm{E}-3$ & $0.490255707173 \mathrm{E}-1,-0.933810555325 \mathrm{E}-1$ \\
\hline 0.999 & $-0.779612000864,1.57384208867$ & $99.5783633460,-218.316883034$ \\
& $-0.779612000864,1.57384208867$ & $99.5783633453,-218.316883034$ \\
\hline \hline & $\sqrt{s}=79.403 \mathrm{GeV}$ & $\sqrt{s}=81.403 \mathrm{GeV}$ \\
\hline \multirow{2}{*}{0.999} & $0.227686132083 \mathrm{E}-4,-0.303846732065 \mathrm{E}-4$ & $0.243884328473 \mathrm{E}-4,-0.327139389502 \mathrm{E}-4$ \\
& $0.227686132074 \mathrm{E}-4,-0.303846732065 \mathrm{E}-4$ & $0.243884328481 \mathrm{E}-4,-0.327139389502 \mathrm{E}-4$ \\
\hline 0 & $-0.504773282953 \mathrm{E}-3,0.120287840705 \mathrm{E}-3$ & $-0.507195719751 \mathrm{E}-3,-0.248876535390 \mathrm{E}-3$ \\
& $-0.504773282955 \mathrm{E}-3,0.120287840705 \mathrm{E}-3$ & $-0.507195719749 \mathrm{E}-3,-0.248876535390 \mathrm{E}-3$ \\
\hline 0.999 & $-5.59686285604,1.92566453330$ & $-5.65305814019,-2.07873259553$ \\
& $-5.59686285604,1.92566453330$ & $-5.65305814019,-2.07873259553$ \\
\hline \hline
\end{tabular}

As is seen from the Table, there is agreement within 9-12 digits for real parts and within 12 digits for imaginary parts, which seems quite satisfactory given that we are using only Double Precision in the Fortran code. We also note that exact formulae with extremely small values of masses $m_{\gamma}, m_{1}, m_{4}$ together with large masses $m_{2}, m_{3}$ occur in LoopTools which could result in loss of computational precision. Unlike LoopTools, the formulae derived in this paper are rather compact and do not explicitly contain the masses $m_{\gamma}, m_{1}, m_{4}$; the latter 
property is the main goal of this paper. Also, the short formulae of this paper demonstrate the underlying physics of singularity cancellations and, moreover, their execution is much faster than LoopTools. On the other hand, one may also conclude that this comparison proves the high reliability of the LoopTools package.

\subsubsection{The function $J_{\text {subsub,2 }}^{\text {tbud }}$}

This function is derived by means of simply setting $m_{2}=0$ in Eq. (88). Let,

$$
J_{\text {subsub }, 2}^{\text {tbud }}=J_{0}^{0}+J_{1}^{0}+J_{2}^{0} .
$$

Since $J_{s u b, 0}^{t b u d}$ is independent of $m_{2}$, we have

$$
J_{0}^{0}=J_{s u b, 0}^{t b u d},
$$

with the latter given by Eq. (88)

For the part $J_{1}^{0}$ one gets:

$$
\begin{aligned}
J_{1}^{0}= & \ln \left(R_{13}\right) \ln \left(\frac{T^{2}+\Delta_{13}+i \epsilon}{\Delta_{13}}\right) \\
& +\operatorname{Li}_{2}\left(\frac{T^{2}}{T^{2}+m_{1}^{2}}\right)-\operatorname{Li}_{2}\left[\left(-\frac{T^{2}}{\Delta_{13}}-i \epsilon\right) \frac{1}{R_{13}}\right]+\operatorname{Li}_{2}\left(-\frac{T^{2}}{\Delta_{13}}-i \epsilon\right) .
\end{aligned}
$$

For the second part, $J_{2}^{0}$, we introduce some common notation:

$$
\begin{aligned}
t^{\max } & =\frac{1}{y^{\max }}\left[\sqrt{T^{4}\left(y^{\max }\right)^{2}+2 T^{2} y^{\max } \sqrt{C_{2}}+C_{2}}-\sqrt{C_{2}}\right], \\
y^{\max } & =-\frac{m_{1}^{2}-i \epsilon}{T^{2}}, \quad y^{\min }=-1-\frac{m_{1}^{2}-i \epsilon}{T^{2}} \\
\sqrt{C_{2}} & =m_{3}^{2}-i \epsilon .
\end{aligned}
$$

There are two solutions:

- if $T^{2}+m_{1}^{2}-m_{3}^{2} \geq 0$, then

$$
\begin{aligned}
J_{2}^{0}= & \frac{1}{2\left(T^{2}+m_{1}^{2}\right)}\left\{\left[\ln \left(y_{l-} \frac{D_{t}}{T^{2}}\left(1-\frac{y_{l+}}{y_{l-}}\right)\right)-\ln \left(-y_{l+} \frac{D_{t}}{T^{2}}\right)-\ln \left(1-\frac{y_{l-}}{y_{l+}}\right)\right] l\left(y_{l-}\right)\right. \\
& \left.+\frac{1}{2} l^{2}\left(y_{l+}\right)-\ln \left(\frac{2 \sqrt{C_{2}}}{y_{l+} D_{t}}\right) l\left(y_{l+}\right)+\operatorname{Li}_{2}\left(\frac{1-y_{l-}}{y_{l+}-y_{l-}}\right)-\operatorname{Li}_{2}\left(\frac{-y_{l-}}{y_{l+}-y_{l-}}\right)\right\},
\end{aligned}
$$

where

$$
\begin{aligned}
t^{\min } & =-T^{2}-\frac{2\left(m_{3}^{2}-i \epsilon\right)}{y^{\min }} \\
D_{t} & =t^{\max }-t^{\min } \\
y_{l \mp} & = \pm \frac{T^{2} \mp t^{\min }}{D_{t}}
\end{aligned}
$$


- if $T^{2}+m_{1}^{2}-m_{3}^{2} \leq 0$, then

$$
\begin{aligned}
J_{2}^{0}= & \frac{1}{2\left(T^{2}+m_{1}^{2}\right)}\left\{\frac{1}{2} \ln ^{2}\left(\frac{D_{t}}{2 T^{2}}\right)-\frac{1}{2} \ln ^{2}\left(-\frac{D_{t} \sqrt{C_{2}}}{2 T^{2}\left(y^{\min } T^{2}+\sqrt{C_{2}}\right)}\right)\right. \\
& +\ln \left(\frac{2 \sqrt{C_{2}}}{y_{l+} D_{t}}\right) \ln \left(-\frac{D_{t} \sqrt{C_{2}}}{y^{\min }\left(T^{2}\right)^{2}}\right)-\ln \left(-\frac{2 y^{\min } T^{2}}{D_{t} y_{l+}}\right) \ln \left(\frac{D_{t}}{2 T^{2}}\right) \\
& -\ln \left(1+\frac{\sqrt{C_{2}}}{y^{\min ^{2}}}\right) \ln \left(\frac{D_{t}}{2 T^{2}}\right)-\ln \left(\frac{2 \sqrt{C_{2}}}{y_{l+} D_{t}}\right) l\left(y_{l+}\right) \\
& \left.+\frac{1}{2} l^{2}\left(y_{l+}\right)+\operatorname{Li}_{2}\left(\frac{1}{y_{l+}}\right)-\operatorname{Li}_{2}\left(\frac{\sqrt{C_{2}}}{y^{\min } T^{2}+\sqrt{C_{2}}}\right)-\zeta(2)\right\},
\end{aligned}
$$

with

$$
\begin{aligned}
t^{\min } & =T^{2} \\
D_{t} & =t^{\max }-t^{\min }, \\
y_{l+} & =-\frac{2 T^{2}}{D_{t}} .
\end{aligned}
$$

In Table 2 we give a similar comparison as in Table 1 but now it is for the function $J_{\text {subsub }, 2}^{\text {tbud }}$ defined by Eq. (100) and related ones. The setup is the same as for Table 1. Again, first lines — LoopTools, second lines — this paper.

Table 2: Comparison of $J_{\text {subsub }, 2}^{\text {tbud }}$

\begin{tabular}{||c|c|c||}
\hline \hline $\cos \theta$ & $\sqrt{s}=1 \mathrm{GeV}$ & $\sqrt{s}=173.2 \mathrm{GeV}$ \\
\hline-0.999 & $-0.943679024194 \mathrm{E}-3,0$ & $-0.495811769871 \mathrm{E}-6,0.120460853637 \mathrm{E}-5$ \\
& $-0.943679024191 \mathrm{E}-3,0$ & $-0.495811769847 \mathrm{E}-6,0.120460853637 \mathrm{E}-5$ \\
\hline 0 & $-0.177753225378 \mathrm{E}-3,0$ & $-0.373359924505 \mathrm{E}-6,-0.345101621985 \mathrm{E}-8$ \\
& $-0.177753225357 \mathrm{E}-3,0$ & $-0.373359924465 \mathrm{E}-6,-0.345101621921 \mathrm{E}-8$ \\
\hline 0.999 & $-0.128202726721 \mathrm{E}-3,0$ & $-0.253792663608 \mathrm{E}-6,-0.119084548438 \mathrm{E}-5$ \\
$\operatorname{SANC}=$ & $-0.128202726700 \mathrm{E}-30$ & $-0.253792663600 \mathrm{E}-6,-0.119084548438 \mathrm{E}-5$ \\
\hline \hline & $\sqrt{s}=79.403 \mathrm{GeV}$ & $\sqrt{s}=81.403 \mathrm{GeV}$ \\
\hline-0.999 & $0.917891464851 \mathrm{E}-3,0$ & $0.843008928372 \mathrm{E}-3,0.718400159039 \mathrm{E}-3$ \\
& $0.917891464846 \mathrm{E}-3,0$ & $0.843008928376 \mathrm{E}-3,0.718400159039 \mathrm{E}-3$ \\
\hline 0 & $-0.188317064868 \mathrm{E}-3,0$ & $-0.177932415951 \mathrm{E}-3,-0.901230697461 \mathrm{E}-4$ \\
& $-0.188317064870 \mathrm{E}-3,0$ & $-0.177932415950 \mathrm{E}-3,-0.901230697461 \mathrm{E}-4$ \\
\hline 0.999 & $-0.266362647090 \mathrm{E}-3,0$ & $-0.258030469532 \mathrm{E}-3,-0.157505612576 \mathrm{E}-3$ \\
& $-0.266362647091 \mathrm{E}-3,0$ & $-0.258030469531 \mathrm{E}-3,-0.157505612576 \mathrm{E}-3$ \\
\hline \hline
\end{tabular}

As seen from the Table, there is again agreement within 10-12 digits for real and imaginary parts. Below the $W$ resonance this function is real.

We switch now to another example of $J$ functions. 


\section{Calculation of $J$ for the process $u d \rightarrow t b$}

As in the previous section we shall consider only the direct and $\gamma W$ diagrams. For the process $u d \rightarrow t b$ the list of arguments of the universal function $J$ is: $m_{1}^{2}=m_{b}^{2}, m_{2}^{2}=m_{t}^{2}, m_{3}^{2}=M_{W}^{2}$, $m_{4}^{2}=m_{q}-$ singular mass.

$$
J \equiv J_{A W}^{d}\left(Q^{2}, T^{2} ; m_{d}, m_{u}, m_{b}, m_{t}\right)
$$

In the following presentation we change the notation of masses as in (13) and neglect the mass $m_{u}$ which does not lead to a mass singularity.

\subsection{Integration with respect to $z, x$}

We omit the details of the integrations with respect to $z$ and to $x$. The standard ingredients are:

$$
\begin{aligned}
N_{x y} & =-2 k_{x y} p_{2}=N_{y}, \\
N_{y} & =T^{2}(1-y)+Q^{2}+m_{2}^{2}(1-y)+m_{4}^{2} \\
k_{x y}^{2} & =N_{y} x-T_{y}^{2}, \\
T_{y}^{2} & =T^{2} y(1-y)+m_{1}^{2}(1-y)+m_{4}^{2} y, \\
L & =P^{2} x-i \epsilon .
\end{aligned}
$$

Contrary to the case of $t \rightarrow$ bud decay, the variables $k_{x y}^{2}$ and $L^{*}=L-k_{x y}^{2}$ are linear in $x$ in this case since we have neglected $m_{u}$ :

$$
L^{*}=L-k_{x y}^{2}=\left[-\left(T^{2}+m_{2}^{2}\right)(1-y)+m_{3}^{2}-m_{4}^{2}\right] x+T_{y}^{2}-i \epsilon .
$$

\subsection{Integration over $y$}

The one-dimensional integral with respect to $y \rightarrow(1-y)$ is

$$
J=\int_{0}^{1} d y I(y)
$$

With the aid of Eq. (27) and with ingredients of Eqs. (109 110) for the integrand $I(y)$ one obtains a simpler result than Eqs. (29 30):

$$
I(y)=\left(-\frac{1}{k_{x y \mid y}^{2}}-\frac{1}{T_{y}^{2}-i \epsilon}\right)\left[\ln \left(L_{\mid y}^{*}\right)-\ln \left(P^{2} y\right)\right] .
$$

where

$$
\begin{aligned}
P^{2} & =Q^{2}+m_{3}^{2}-i \epsilon, \\
T_{y}^{2} & =T^{2} y(1-y)+m_{1}^{2} y+m_{4}^{2}(1-y), \\
k_{x y \mid y}^{2} & =m_{2}^{2} y(1-y)-m_{1}^{2} y+Q^{2}(1-y), \\
L_{\left.\right|_{y}}^{*} & =-m_{2}^{2} y(1-y)+m_{1}^{2} y+m_{3}^{2}(1-y)-i \epsilon .
\end{aligned}
$$




\subsubsection{Integrand in the limit $m_{4} \rightarrow 0$}

After some algebra one can derive the following expression for $I(y)$ valid in the limit $m_{4} \rightarrow 0$ :

$$
I(y)=\frac{1}{\sqrt{D_{k}}}\left[\mathcal{T}\left(y, y_{k_{1}}\right)-\mathcal{T}\left(y, y_{k_{2}}\right)\right]+\frac{1}{\left(T^{2}+m_{1}^{2}\right)}\left[\mathcal{T}\left(y, y_{1}\right)-\mathcal{T}\left(y, y_{2}\right)\right]
$$

Thus $I(y)$ is expressed in terms of the auxiliary function

$$
\mathcal{T}(y, \eta)=\frac{1}{(y-\eta)}\left[\ln \left(1-\frac{y}{y_{L_{1}^{*}}}\right)+\ln \left(1-\frac{y}{y_{L_{2}^{*}}}\right)-\ln (1-y)-\ln \left(\frac{P^{2}}{m_{3}^{2}}\right)\right]
$$

with roots of the quadratic trinomial $T_{y}^{2}-i \epsilon$ :

$$
y_{1}=\frac{T^{2}+m_{1}^{2}-i \epsilon}{T^{2}}, \quad y_{2}=-\frac{m_{4}^{2}-i \epsilon}{T^{2}+m_{1}^{2}-i \epsilon} .
$$

The other objects arise from the trinomial $k_{x y \mid y}^{2}$ in $y$

$$
\begin{aligned}
k_{x y \mid y}^{2} & =-m_{2}^{2} y^{2}+\left(\Delta_{21}-Q^{2}\right) y+\left(Q^{2}+i \epsilon\right) \\
B_{k} & =\Delta_{21}-Q^{2} \\
\sqrt{D_{k}} & =\sqrt{B_{k}^{2}+4 m_{2}^{2}\left(Q^{2}+i \epsilon\right)}
\end{aligned}
$$

with roots:

$$
y_{k_{1}}=\frac{-B_{k}+\sqrt{D_{k}}}{2\left(-m_{2}^{2}\right)}, \quad y_{k_{2}}=\frac{-B_{k}-\sqrt{D_{k}}}{2\left(-m_{2}^{2}\right)}
$$

and trinomial $\left.L^{*}\right|_{y}$ in $y$, i.e.

$$
\begin{aligned}
L_{\mid y}^{*} & =m_{2}^{2} y^{2}+y\left(\Delta_{12}-m_{3}^{2}\right)+\left(m_{3}^{2}-i \epsilon\right) \\
B_{L_{l}^{*}} & =\Delta_{12}-m_{3}^{2} \\
\sqrt{D_{L_{l}^{*}}} & =\sqrt{B_{L_{l}^{*}}^{2}-4 m_{2}^{2}\left(m_{3}^{2}-i \epsilon\right)}
\end{aligned}
$$

with roots:

$$
y_{L_{1}^{*}}=\frac{-B_{L_{l}^{*}}+\sqrt{D_{L_{l}^{*}}}}{2 m_{2}^{2}}, \quad y_{L_{2}^{*}}=\frac{-B_{L_{l}^{*}}-\sqrt{D_{L_{l}^{*}}}}{2 m_{2}^{2}} .
$$

\subsection{Function $J$}

The final answer for the integral $J$ of Eq. (111) is split into two parts,

$$
J=J_{0}+J_{a d d}
$$


The first part, free of $m_{4}^{2}$ mass singularity, is

$$
\begin{aligned}
J_{0}= & \frac{1}{\sqrt{D_{k}}}\left[\left\{\left[\ln \left(1-y_{k_{1}}\right)+\ln \left(R_{3}\right)\right] l\left(y_{k_{1}}\right)-\mathcal{M}\left(y_{L_{1}^{*}}, y_{k 1}\right)-\mathcal{M}\left(y_{L_{2}^{*}}, y_{k 1}\right)\right.\right. \\
& \left.\left.+\operatorname{Li}_{2}\left(-\frac{y_{k_{1}}}{1-y_{k_{1}}}\right)\right\}-\left\{y_{k_{1}} \rightarrow y_{k_{2}}\right\}\right] \\
& +\frac{1}{T^{2}+m_{1}^{2}}\left[\left\{\mathcal{M}\left(y_{L_{1}^{*}}, y_{1}\right)+\operatorname{Li}_{2}\left(\frac{1}{y_{L_{1}^{*}}}\right)\right\}+\left\{y_{L_{1}^{*}} \rightarrow y_{L_{2}^{*}}\right\}\right. \\
& \left.-\left[\ln \left(1-y_{1}\right)+\ln \left(R_{3}\right)\right] l\left(y_{1}\right)-\operatorname{Li}_{2}\left(-\frac{y_{1}}{1-y_{1}}\right)\right],
\end{aligned}
$$

and the additional part that depends on $m_{4}^{2}$ :

$$
J^{a d d}=\frac{1}{T^{2}+m_{1}^{2}} \ln \left(R_{3}\right) \ln \left(R_{14}\right)
$$

For simplicity we introduce the notation

$$
R_{14}=\frac{T^{2}+m_{1}^{2}-i \epsilon}{m_{4}^{2}}
$$

\subsection{Subtracted function $J_{s u b}^{u d t b}$}

The subtracted function $J_{s u b}^{u d t b}$ is defined by the following equation (see Ref. [4]):

$$
J_{\text {sub }}^{u d t b}=J-\frac{Q^{2}}{T^{2}+m_{1}^{2}} C_{0}\left(0, m_{4}^{2}, Q^{2} ; m_{3}, m_{4}, 0\right) .
$$

After adding to this the mass singular $C_{0}$ function, we get the following expression free of $m_{4}$ mass singularity:

$$
J_{s u b}^{u d t b}=J_{0}+\ln \left(R_{3}\right)\left[\ln \left(-\frac{Q^{2}}{m_{3}^{2}}\right)+\ln \left(R_{13}\right)\right]-\operatorname{Li}_{2}\left(R_{3}\right)+\zeta(2),
$$

where $R_{3}$ and $R_{13}$ are defined by Eqs. (87,41) respectively.

\subsubsection{Second substraction $J_{\text {subsub, } 1}^{u d t b}$}

A second substraction eliminates the $b$ quark mass singularity:

$$
\begin{aligned}
J_{\text {subsub }, 1}^{u d t b}\left(Q^{2}, T^{2} ; m_{b}, m_{t}, M_{W}\right)= & J_{s u b, 1}^{u d t b}\left(Q^{2}, T^{2} ; m_{b}, m_{t}, M_{W}\right) \\
& -\frac{\left(Q^{2}+T^{2}+m_{t}^{2}\right)}{T^{2}} C_{0}\left(-m_{t}^{2},-m_{b}^{2}, Q^{2} ; M_{W}, m_{b}, 0\right) .
\end{aligned}
$$


The limit in $m_{b}$ exists and reads

$$
\begin{aligned}
J_{\text {subsub }, 1}^{u d t b}= & \frac{1}{T^{2}}\left[-\ln \left(R_{3}\right) \ln \left(\frac{P}{T^{2}}\right)+\ln \left(-\frac{P}{\Delta_{23}}\right) \ln \left(\frac{\left(Q^{2}+m_{3}^{2}\right)^{2}}{\left(Q^{2}+m_{2}^{2}\right)^{2}} \frac{T^{2}}{m_{3}^{2}}\right)\right. \\
& \left.+2 \operatorname{Li}_{2}\left(-\frac{\Delta_{23}}{P}\right)+\operatorname{Li}_{2}\left(1+r_{23}\right)-\operatorname{Li}_{2}\left(\frac{m_{2}^{2}}{\Delta_{23}+i \epsilon}\right)-3 \zeta(2)\right]
\end{aligned}
$$

with

$$
r_{i 3}=\frac{T^{2}}{m_{i}^{2}-m_{3}^{2}+i \epsilon} .
$$

See in this case Eq. (13) for the meaning of $m_{i}$.

In Table 3 we give the comparison of real and imaginary parts of the function $J_{\text {subsub, } 1}^{u d t b}$ defined by Eq. (127) and related ones, computed using the LoopTools package with numbers, derived exactly from Eq. (128), i.e. with results of this paper. The numbers are given for two values of variable $s$ (near threshold and at a high value), for three values of $\cos \theta$. The masses are $m_{\gamma}=10^{-40}, m_{1}=10^{-8}, m_{2}=174.3, m_{3}=80.403, m_{4}=10^{-7}$ (all masses are given in $\mathrm{GeV}$ ); first lines — LoopTools, second lines — this paper.

Table 3: Comparison of $J_{\text {subsub }, 1}^{u d t b}$

\begin{tabular}{||c|cc|c||}
\hline \hline $\cos \theta$ & \multicolumn{2}{|c|}{$\sqrt{s}=200 \mathrm{GeV}$} & $\sqrt{s}=10^{4} \mathrm{GeV}$ \\
\hline \multirow{2}{*}{0.999} & -2.07194259988, & 7.01180433443 & $-0.102517409116 \mathrm{E}-2,0.102816191182 \mathrm{E}-2$ \\
& -2.07194259988, & 7.01180433443 & $-0.102517409116 \mathrm{E}-2,0.102816191182 \mathrm{E}-2$ \\
\hline 0 & $0.878509046507 \mathrm{E}-3$, & $0.238029806504 \mathrm{E}-02$ & $0.895389809985 \mathrm{E}-6,0.184388800385 \mathrm{E}-6$ \\
& $0.878509046507 \mathrm{E}-3$, & $0.238029806504 \mathrm{E}-02$ & $0.895389809985 \mathrm{E}-6,0.184388800385 \mathrm{E}-6$ \\
\hline 0.999 & $0.602158734688 \mathrm{E}-3$, & $0.913885117129 \mathrm{E}-03$ & $0.517213769582 \mathrm{E}-6,0.486927343347 \mathrm{E}-7$ \\
& $0.602158734687 \mathrm{E}-3$, & $0.913885117129 \mathrm{E}-03$ & $0.517213769582 \mathrm{E}-6,0.486927343347 \mathrm{E}-7$ \\
\hline \hline
\end{tabular}

As is seen from this Table, there is agreement within 11-12 digits for the real parts and within 12 digits for imaginary parts. We note again that Eq. (128) derived in this paper is very compact and does not contain the masses $m_{\gamma}, m_{1}, m_{4}$ explicitly.

\subsubsection{No second subtraction $J_{\text {subsub,2 }}^{u d t b}$}

The function $J_{\text {subsub, } 2}^{u d t b}\left(Q^{2}, T^{2} ; m_{t}, m_{b}, M_{W}\right)$ with exchanged arguments has no $m_{b}$ mass singularity. Since we need no limit in $m_{t}$, we just rename the second function, assuming that $m_{b}$ is set to zero:

$$
J_{\text {subsub }, 2}^{u d t b}\left(Q^{2}, T^{2} ; m_{t}, m_{b}, M_{W}\right)=J_{\text {sub }}^{u d t b}\left(Q^{2}, T^{2} ; m_{t}, m_{b}, M_{W}\right) .
$$


The answer is found straightforwardly from Eq. (126) for $J_{\text {sub }}^{u d t b}$ and $J_{0}$ from Eq. (122) with interchanged arguments $m_{1}=m_{t}, m_{2}=m_{b}=0$ :

$$
\begin{aligned}
J_{\text {subsub }, 2}^{u d t b}= & \frac{1}{Q^{2}+m_{1}^{2}}\left[+\operatorname{Li}_{2}\left(\frac{Q^{2}}{m_{1}^{2}} \frac{\Delta_{13}}{P^{2}}\right)-\operatorname{Li}_{2}\left(-\frac{\Delta_{13}}{P^{2}}\right)-\operatorname{Li}_{2}\left(-\frac{Q^{2}}{m_{1}^{2}-i \epsilon}\right)+\zeta(2)\right] \\
& +\frac{1}{T^{2}+m_{1}^{2}}\left[-\ln \left(-\frac{P^{2}}{T^{2}+\Delta_{13}}\right) \ln \left(\frac{m_{1}^{2}}{T^{2}+m_{1}^{2}}\right)-\ln \left(R_{3}\right) \ln \left(-\frac{Q^{2}}{T^{2}+m_{1}^{2}}\right)\right. \\
& +\operatorname{Li}_{2}\left(-\frac{\Delta_{13}}{m_{3}^{2}-i \epsilon}\right)-\operatorname{Li}_{2}\left(R_{3}\right)-\operatorname{Li}_{2}\left(\frac{T^{2}+m_{1}^{2}}{m_{1}^{2}-i \epsilon}\right) \\
& \left.+\operatorname{Li}_{2}\left(\frac{T^{2}+m_{1}^{2}}{m_{1}^{2}} \frac{1}{1+r_{13}}\right)-\operatorname{Li}_{2}\left(\frac{1}{1+r_{13}}\right)+\zeta(2)\right] .
\end{aligned}
$$

The meaning of $m_{3}=M_{W}$ remains unchanged.

In Table 4 we give a similar comparison as in Table 3 but now for the function $J_{\text {subsub,2 }}^{u d t b}$

\begin{tabular}{|c|c|c|}
\hline $\cos \theta$ & $\overline{\sqrt{s}}=200 \mathrm{GeV}$ & $\sqrt{s}=10^{4} \mathrm{GeV}$ \\
\hline \multirow{2}{*}{-0.999} & $0.159231105282 \mathrm{E}-4,0.118244938272 \mathrm{E}-3$ & $-0.195869068435 \mathrm{E}-3 \quad 0.240805881821 \mathrm{E}-3$ \\
\hline & $0.159231105282 \mathrm{E}-4,0.118244938272 \mathrm{E}-3$ & $-0.195869068435 \mathrm{E}-3 \quad 0.240805881821 \mathrm{E}-3$ \\
\hline \multirow[t]{2}{*}{0} & $0.207059680614 \mathrm{E}-4,0.881532449471 \mathrm{E}-4$ & $0.110155292282 \mathrm{E}-5-0.167170803336 \mathrm{E}-6$ \\
\hline & $0.207059680614 \mathrm{E}-4,0.881532449471 \mathrm{E}-4$ & $0.110155292282 \mathrm{E}-5-0.167170803336 \mathrm{E}-6$ \\
\hline \multirow[t]{2}{*}{0.999} & $0.234082370485 \mathrm{E}-4,0.682484139707 \mathrm{E}-4$ & $0.459502689190 \mathrm{E}-6-0.184916952415 \mathrm{E}-10$ \\
\hline & $0.234082370486 \mathrm{E}-4,0.682484139707 \mathrm{E}-4$ & $0.459502689190 \mathrm{E}-6-0.184916952411 \mathrm{E}-10$ \\
\hline
\end{tabular}
defined by Eq. (130) and the related ones. The setup is the same as for Table 1, Again, first lines - LoopTools, second lines — this paper.

Table 4: Comparison of $J_{\text {subsub,2 }}^{u d t b}$

As is seen from this Table, there is again agreement within 11-12 digits for real and imaginary parts.

\section{Functions $J$ for the process $b u \rightarrow t d$}

In this section we briefly consider $J$ functions arising in four $b u \rightarrow t d$ processes. If one neglects the $b$ quark mass, there appear only four doubly subtracted $J_{\text {subsub }}^{\text {butd }}$ functions, see [4]:

$$
\begin{aligned}
& J_{\text {subsub, } 1}^{\text {butd }}\left(Q^{2}, T^{2}, 0, m_{t}, M_{W}\right), \\
& J_{\text {subsub }, 1}^{\text {butd }}\left(Q^{2}, U^{2}, 0, m_{t}, M_{W}\right), \\
& J_{\text {subsub }, 2}^{\text {butd }}\left(Q^{2}, T^{2}, m_{t}, 0, M_{W}\right), \\
& J_{\text {subsub }, 2}^{\text {butd }}\left(Q^{2}, U^{2}, m_{t}, 0, M_{W}\right) .
\end{aligned}
$$

For four possible channels, one has the following correspondence between arguments $Q^{2}, T^{2}, U^{2}$ and $s, \cos \theta$, where always $\theta=L\left(\vec{p}_{2}, \vec{p}_{3}\right)$ (see 4-momenta assignment below): 
1. $b\left(p_{1}\right)+u\left(p_{2}\right) \rightarrow d\left(p_{3}\right)+t\left(p_{4}\right)$

$$
Q^{2}=\frac{s-m_{t}^{2}}{2} c_{-}=-t, \quad T^{2}=\frac{s-m_{t}^{2}}{2} c_{+}=-u, \quad U^{2}=-s,
$$

2. $b\left(p_{1}\right)+\bar{d}\left(p_{2}\right) \rightarrow \bar{u}\left(p_{3}\right)+t\left(p_{4}\right)$

$$
Q^{2}=\frac{s-m_{t}^{2}}{2} c_{-}=-t, \quad T^{2}=-s, \quad U^{2}=\frac{s-m_{t}^{2}}{2} c_{+}=-u,
$$

3. $\bar{b}\left(p_{1}\right)+\bar{u}\left(p_{2}\right) \rightarrow \bar{d}\left(p_{3}\right)+\bar{t}\left(p_{4}\right)$

$$
Q^{2}=\frac{s-m_{t}^{2}}{2} c_{-}=-t, \quad T^{2}=\frac{s-m_{t}^{2}}{2} c_{+}=-u, \quad U^{2}=-s,
$$

4. $\bar{b}\left(p_{1}\right)+d\left(p_{2}\right) \rightarrow u\left(p_{3}\right)+\bar{t}\left(p_{4}\right)$

$$
Q^{2}=\frac{s-m_{t}^{2}}{2} c_{-}=-t, \quad T^{2}=-s \quad U^{2}=\frac{s-m_{t}^{2}}{2} c_{+}=-u,
$$

where

$$
c_{ \pm}=1 \pm \cos \theta \text {. }
$$

There is an important difference between $J$ functions considered in Sections 2 and 3 and in this Section. In the first two cases two Mandelstam variables $T^{2}=-t$ and $U^{2}=-u$ have the same sign; they are different only by the $\operatorname{sign}$ of $\cos \theta$. In this, third case, $Q^{2}$ has always the sense of $-t$, while $T^{2}$ and $U^{2}$ change their meaning $-u$ or $-s$ and therefore the sign. This is the reason why two $t$ channel $J$ 's, for which both arguments are positive, can not be computed using the $J$ functions derived for channels considered previously, and the calculation for such sign assignments has to be repeated from scratch. This task is beyond the scope of this paper and will be presented elsewhere. For the time being we will use a pragmatic solution. We noted that equations of Section 2 give correct answers for the case $Q^{2}>0$ and $T^{2}<0$ or $U^{2}<0$.

Table 5: Comparison of $J_{\text {subsub, } 1}^{\text {butd,- }}$

\begin{tabular}{||c|c|c||}
\hline \hline $\cos \theta$ & $\sqrt{s}=200 \mathrm{GeV}$ & $\sqrt{s}=10^{4} \mathrm{GeV}$ \\
\hline-0.999 & $-0.173090211233 \mathrm{E}-04,0.216758293310 \mathrm{E}-04$ & $-0.467497992886 \mathrm{E}-06,0.254446155152 \mathrm{E}-06$ \\
& $-0.173090211232 \mathrm{E}-04,0.216758293312 \mathrm{E}-04$ & $-0.467497992836 \mathrm{E}-06,0.254446155157 \mathrm{E}-06$ \\
\hline 0 & $-0.451130695567 \mathrm{E}-05,0.149343040736 \mathrm{E}-05$ & $-0.462653095799 \mathrm{E}-06,0.210944911427 \mathrm{E}-06$ \\
& $-0.451130695569 \mathrm{E}-05,0.149343040746 \mathrm{E}-05$ & $-0.462653095748 \mathrm{E}-06,0.210944911431 \mathrm{E}-06$ \\
\hline 0.999 & $0.172894787644 \mathrm{E}-04,-0.216698015838 \mathrm{E}-04$ & $0.105671318711 \mathrm{E}-06,-0.193311004466 \mathrm{E}-06$ \\
& $0.172894787643 \mathrm{E}-04,-0.216698015840 \mathrm{E}-04$ & $0.105671318762 \mathrm{E}-06,-0.193311004461 \mathrm{E}-06$ \\
\hline
\end{tabular}

This is illustrated by Tables 56, where we give a comparison of real and imaginary parts of the function $J_{\text {subsub, } 1}^{\text {butd,- }}$ and $J_{\text {subsub, } 2}^{\text {butd,- }}$ in the $t$ channel, Eq. (132), for the case $Q^{2}>0$ and 
Table 6: Comparison of $J_{\text {subsub, } 2}^{\text {butd,- }}$

\begin{tabular}{||c|c|c||}
\hline \hline $\cos \theta$ & $\sqrt{s}=200 \mathrm{GeV}$ & $\sqrt{s}=10^{4} \mathrm{GeV}$ \\
\hline \multirow{2}{*}{0.999} & $0.108310963092 \mathrm{E}-03,0.297113203560 \mathrm{E}-03$ & $-0.781547133959 \mathrm{E}-06,0.303109233776 \mathrm{E}-06$ \\
& $0.108310963092 \mathrm{E}-03,0.297113203560 \mathrm{E}-03$ & $-0.781547133958 \mathrm{E}-06,0.303109233776 \mathrm{E}-06$ \\
\hline 0 & $0.288401492494 \mathrm{E}-04,0.656706410374 \mathrm{E}-04$ & $-0.723035031957 \mathrm{E}-06,0.259579787125 \mathrm{E}-06$ \\
& $0.288401492495 \mathrm{E}-04,0.656706410373 \mathrm{E}-04$ & $-0.723035031957 \mathrm{E}-06,0.259579787125 \mathrm{E}-06$ \\
\hline 0.999 & $-0.803387429231 \mathrm{E}-04,-0.296822348620 \mathrm{E}-03$ & $0.100393594508 \mathrm{E}-06,-0.166942822727 \mathrm{E}-06$ \\
& $-0.803387429230 \mathrm{E}-04,-0.296822348620 \mathrm{E}-03$ & $0.100393594508 \mathrm{E}-06,-0.166942822727 \mathrm{E}-06$ \\
\hline \hline
\end{tabular}

$T^{2}<0$ or $U^{2}<0$ using the same formulae and setup as for Tables 1 and 2: first lines LoopTools, second lines - this paper.

As is seen from these Tables, there is agreement within 10-11 digits for real and imaginary parts. Note that one can reach agreement to all visible digits with Real*16.

As far as two $t$ channel $J$ 's are concerned, for which both arguments are positive, we accept a temporary solution for the time being, noticing that previously computed $J$ functions return correctly only the real parts. We recall that imaginary parts do not contribute at the one-loop level. The real parts are illustrated by Table 7 .

Table 7: Comparison of $J_{\text {subsub, } 1(2)}^{\text {butd, }}$

\begin{tabular}{||c|c|c||}
\hline \hline $\cos \theta$ & $\sqrt{s}=10^{4} \mathrm{GeV}$ & $\sqrt{s}=10^{4} \mathrm{GeV}$ \\
\hline & $J_{\text {subsub }, 1}^{\text {butd, }}$ & $J_{\text {subsub }, 2}^{\text {butd,+ }}$ \\
\hline-0.999999 & $-0.372108952046 \mathrm{D}+01$ & $-0.122105779653 \mathrm{D}-02$ \\
& $-0.372108952003 \mathrm{D}+01$ & $-0.122105779653 \mathrm{D}-02$ \\
\hline-0.9990 & $-0.122256223637 \mathrm{D}-02$ & $-0.256945712509 \mathrm{D}-03$ \\
& $-0.122256223637 \mathrm{D}-02$ & $-0.256945712509 \mathrm{D}-03$ \\
\hline 0 & $0.707331687150 \mathrm{D}-06$ & $0.122739516453 \mathrm{D}-05$ \\
& $0.707331687129 \mathrm{D}-06$ & $0.122739516453 \mathrm{D}-05$ \\
\hline 0.9999 & $-0.155145904970 \mathrm{D}-06$ & $-0.149761802457 \mathrm{D}-06$ \\
& $-0.155145905078 \mathrm{D}-06$ & $-0.149761802457 \mathrm{D}-06$ \\
\hline 0.99999 & $-0.515690518852 \mathrm{D}-06$ & $-0.496884568518 \mathrm{D}-06$ \\
& $-0.515690519134 \mathrm{D}-06$ & $-0.496884568519 \mathrm{D}-06$ \\
\hline \hline
\end{tabular}

As is seen from these Tables, there is an agreement within 9-12 digits for real parts and that the agreement does not improve with Real*16 computations.

\section{$5 \quad$ SANC packages}

The numeric comparison with the LoopTools library presented in this paper can be verified with help of the SANC software packages. We have three packages related to the $J_{\text {subsub,1(2) }}$ functions for three channels. They are available to download from the web pages of SANC project [6]. 


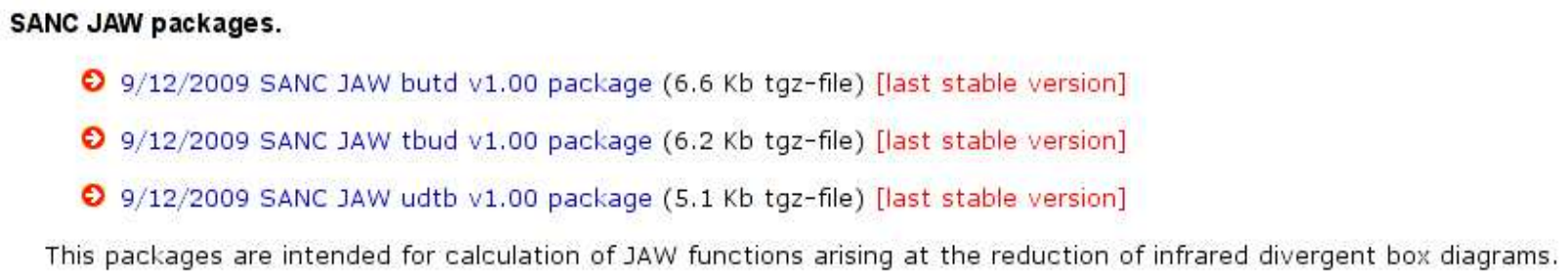

Figure 2: SANC packages download page

Each package contains the following files:

- main files for both $J_{\text {subsub,1(2) }}$ functions;

- $J_{\text {subsub,1(2) }}$ source files;

- utility functions;

- Makefile;

- README, INSTALL, LICENSE and other information files.

\section{Conclusions}

The invention and usage of a new class of functions $J$, relevant to the Passarino-Veltman reduction [1], has become a standard step in the chain of calculations in project SANC. Originally they were introduced in [3].

Obvious advantages and disadvantages of these functions are:

1. final results are compact and evidently demonstrate the fundamentals of physics;

2. the subtracted $J_{W A \text {,sub }}$ functions have no mass singularities;

3. their compactness results in stable and very rapid calculations;

4. the functions of this new class are linear combinations of the standard Passarino-Veltman $D_{0}$ and $C_{0}$ functions and their explicit form depends on the concrete channel of a process; namely, they depend of the choice of channel because we have no universal expressions for the $J$ functions,

5. for the analytic calculations we have, nevertheless, a consistent method;

6. it would be desirable to improve the way of the analytic calculations in order to obtain various channels by a simple rotation of their arguments, as is done in the LoopTools package for $D_{0}$ functions. 


\section{Acknowledgement}

We are indebted to S.G. Bondarenko for useful discussions. This paper is partly supported by grant RFFI $N^{o}$ 07-02-00932.

\section{References}

[1] G. Passarino and M. J. G. Veltman, Nucl. Phys. B160 (1979) 151.

[2] D. Y. Bardin and G. Passarino, Oxford, UK: Clarendon (1999) 685 p.

[3] D. Y. Bardin, L. V. Kalinovskaya, and L. A. Rumyantsev, Phys. Part. Nucl. Lett. 6 (2009) $30-41$.

[4] D. Bardin et al., Phys. Part. Nucl. Lett. 7 (2010) 128-141, 0903.1533 [hep-ph].

[5] T. Hahn and M. Perez-Victoria, Comput. Phys. Commun. 118 (1999) 153-165, hep-ph/9807565.

[6] Dubna - http://sanc.jinr.ru, CERN - http://pcphsanc.cern.ch (2007). 